\title{
Metabolic Rather than Pathological Response to Preoperative Chemoradiotherapy Is a Stronger Predictor of Survival in cStage IIB-IV Esophageal Cancer
}

\author{
SATORU MOTOYAMA ${ }^{1}$, YUSUKE SATO ${ }^{1}$, KIYOTOMI MARUYAMA $^{1}$, SHUETSU USAMI $^{1}$, \\ KEI YOSHINO ${ }^{1}$, TOSHINOBU NAKATSU ${ }^{1}$, TOMOHIKO SASAKI $^{1}$, AKIYUKI WAKITA $^{1}$, YUTA KAWAKITA $^{1}$, \\ JIAJIA LIU ${ }^{1}$, AKIRA ANBAI $^{2}$, KOUICHI ISHIYAMA $^{2}$, HAJIME SAITO $^{1}$ and YOSHIHIRO MINAMIYA ${ }^{1}$ \\ ${ }^{1}$ Esophageal Surgery, and ${ }^{2}$ Radiology, Akita University Hospital, Akita, Japan
}

\begin{abstract}
Aim: We investigated which is the stronger predictor, pathological response or metabolic response, for survival outcome in patients treated with neoadjuvant chemoradiotherapy (NACRT) plus esophagectomy for thoracic esophageal squamous cell carcinoma (TESCC). Patients and Methods: Fifty consecutive patients with cStage IIB-IV TESCC were enrolled. We analyzed the pathological response and metabolic response (fractional decrease in tumor maximum standardized uptake value) to NACRT. Independent prognostic factors predictive of 3-year survival were investigated using univariate and multivariate analyses. Results: Among the 50 patients, 10 (20\%) showed a pathological complete response (in both tumor and lymph nodes) and 36 (72\%) showed grade 2-3 pathological response. Univariate analysis showed that age, gender, cT stage, pathological response and metabolic response to be significant prognostic factors. A subsequent multivariate analysis confirmed metabolic response and gender to be significant prognostic factors. Conclusion: Metabolic response for NACRT was an independent prognostic factor and a more powerful predictor of survival compared to pathological response.
\end{abstract}

Esophageal squamous cell carcinoma (SCC) has become the predominant type of esophageal cancer in Asia, Africa, and South America, and among African Americans in North America; it accounts for approximately $90 \%$ of esophageal cancer cases worldwide (1). Recently, multimodal therapy, including chemotherapy, radiotherapy and surgical resection

Correspondence to: Satoru Motoyama, Esophageal Surgery, Akita University Hospital, 1-1-1 Hondo, Akita 010-8543, Japan. Tel: +81 188846132, Fax: +81 188362615, e-mail: motoyama@doc.med.akitau.ac.jp

Key Words: Neoadjuvant chemoradiotherapy, esophagectomy, esophageal cancer, survival, metabolic response. was introduced for treatment of advanced thoracic esophageal squamous cell carcinoma (TESCC), taking advantage of the tumor's high sensitivity to radiotherapy and chemotherapy (2, 3 ). The Cross trial demonstrated that preoperative neoadjuvant chemoradiotherapy (NACRT) followed by esophagectomy achieved both local and systemic control, resulting in improved survival in patients with esophageal cancer. In a subgroup analysis, the pathological complete response (pCR) rate was $49 \%$ among patients with SCC and pathological response to NACRT was a useful predictor for survival outcome (4). In this study, we investigated which is the stronger predictor, pathological or metabolic response, of survival outcome in patients with cStage IIB-IV TESCC with lymph node involvement treated with NACRT followed by esophagectomy with Japanese style extended 3-field lymphadenectomy.

\section{Patients and Methods}

Patients. This study was approved by the Ethics Committee of Akita University Graduate School of Medicine (no. 547). Additional informed consent was obtained from all patients for whom identifying information is included in this article.

The study participants were 50 consecutive Japanese patients treated at Akita University Hospital between April 2009 and January 2014. The patients were treated with preoperative NACRT plus esophagectomy with Japanese style extended 3-field lymphadenectomy for cStage IIB-IV TESSC with either greater than T3 tumor or regional lymph node involvement, including supraclavicular lymph node metastasis, and with Eastern Cooperative Oncology Group performance status (PS) 0-1. For all patients, the esophageal cancer stage, including diagnosis of lymph node metastasis, was determined according to the International Union Against Cancer tumor-node-metastasis (TNM) Classification of Malignant Tumors (seventh edition) (5) at a conference attended by radiologists, physicians and surgeons.

Chemoradiotherapy (NACRT). Chemotherapy consisted of protracted infusion of 5 -fluorouracil $\left(5-\mathrm{FU} ; 800 \mathrm{mg} / \mathrm{m}^{2} /\right.$ day) on days $1-5$ and cisplatin or nedaplatine $\left(80 \mathrm{mg} / \mathrm{m}^{2} /\right.$ day $)$ on day 1 . This protocol was repeated twice with 3 - to 5 -week intervals in 
between. High-energy X-rays (10 MV) were used for radiotherapy. All patients underwent 3-dimensional radiotherapy planning. The gross tumor volume (GTV) was set both in the primary tumor and metastatic lymph nodes. The clinical target volume (CTV) was set using an approximately $1-\mathrm{cm}$ margin around the GTV. The planning target volume was set using a 5$\mathrm{mm}$ margin around the CTV. Concurrent radiotherapy consisted of $2 \mathrm{~Gy} /$ day for 5 days each week, and the total radiation dose was $40 \mathrm{~Gy}$ in 20 fractions.

[ $\left.{ }^{18} \mathrm{~F}\right]$-Fluorodeoxyglucose positron-emission tomography/computed tomography (FDG-PET/CT). Patients were clinically staged using systematic FDG-PET/computed tomographic imaging before NACRT and then 3-4 weeks after NACRT, before surgery. Patients fasted for at least 4 hours before receiving an intravenous injection of $185 \mathrm{MBq} / \mathrm{kg}$ FDG (FDGscan; Nihon Medi-Physics, Tokyo, Japan) and then rested for 1 hour before scanning. All images were acquired using a combined PET/CT scanner (Discovery ST Elite 16; GE Healthcare, Chicago, IL, USA). Low-dose CT images were acquired using a 16-detector row scanner with the following parameters: $120 \mathrm{kV}$, auto $\mathrm{mA}$ (noise index 25.0), $0.6 \mathrm{~s}$ tube rotation, $3.75 \mathrm{~mm}$ section thickness, $512 \times 512$ matrix, $70 \mathrm{~cm}$ field of view. The CT scan was acquired during shallow breathing, and covered an area from the upper thigh to the base of the skull. The maximum standardized uptake value $\left(\mathrm{SUV}_{\max }\right)$ for each patient was established by drawing regions of interest around the primary tumor on attenuation-corrected FDG-PET images and then calculating the value using software integrated into the PET/CT scanner, based on the formula: $\mathrm{SUV}_{\max }=(\mathrm{C}[\mathrm{mCi} / \mathrm{ml}] / \mathrm{ID}[\mathrm{mCi}]) /$ body weight, where $\mathrm{C}$ was the activity at a pixel within the tissue identified by the regions of interest, and ID was the injected dose per kilogram of body weight. We defined $\mathrm{SUV}_{\max }$ as positive when it was 2.5 or more and negative when it was less than 2.5.

Surgery. Esophagectomies were scheduled at more than 3 weeks after completing NACRT, by which time patients had no grade 2 or worse adverse events. Esophagectomy with Japanese style extended 3- field lymph node dissection (bilateral neck, including the supraclavicular lymph nodes, and mediastinal and abdominal lymph nodes) under right thoracotomy was performed as the standard operative method in this study.

Pathological response to NACRT. The pathological response of the primary tumor was graded using the response evaluation criteria for the effects of radiation, chemotherapy, or both published by the Japanese Esophageal Society: 0: no recognized cytological or histological therapeutic effect; 1: slightly effective, with apparently viable cancer cells accounting for at least one-third of the tumor tissue; 2: moderately effective, with viable cancer cells accounting for less than one- third of the tumor tissue; and 3: markedly effective, with no evidence of viable cancer cells $(6,7)$. A pathological complete response (pCR) was considered to have been achieved when no evidence of viable cancer cells was detected in the tumor or lymph nodes.

Evaluations. In this study, we analyzed the pathological and metabolic responses and the 3-year survival rates among patients receiving NACRT followed by esophagectomy. In addition, independent prognostic factors were investigated using univariate and multivariate analyses.
Statistical analysis. Continuous variables are presented as the median (minimum-maximum). Differences between groups were analyzed using Fisher's exact probability test. The survival distribution from the day of initial treatment to 1st January 2017 was determined using the Kaplan-Meier method with the log-rank test. Cox's proportional hazards regression model was used for univariate and multivariate analyses. All statistical analyses were performed using JMP10 (SAS Institute, Cary, NC, USA) and yielded two-sided $p$-values. Values of $p<0.05$ were considered statistically significant.

\section{Results}

The study participants included $41(82 \%)$ males and nine (18\%) females, with an age of 62.5 (rage 43-74) years (Table I). The main tumor characteristics are given in Table I.

Chemotherapy consisting of cisplatin with 5-FU was used for $21(42 \%)$ patients and nedaplatin with 5-FU was used for $29(58 \%)$ patients. The radiotherapy dose was 40 Gy in 47 (94\%) patients, but was less (20-32 Gy) in three patients. NACRT was completed in 37 (74\%) patients. The reason for incomplete of neoadjuvant treatment in 13 patients was bone marrow supression, hyponatremia, renal dysfunction, sepsis, gastric ulcer, osteomyelitis of the mandible and patient wishes.

The duration of the NACRT was 35 (range=11-58) days. The interval between NACRT and esophagectomy was 36 (range $=21-95$ ) days.

Transthoracic esophagectomy was performed with complete resection (R0) in all patients. The operative time was 525 (range $=386-855) \mathrm{min}$, and blood loss was 627 (range=115-3217) $\mathrm{ml}$. The reconstruction consisted of gastric tube pull-up in $41(82 \%)$ patients, while a colonic pedicle was used in nine (18\%) patients. As a route of reconstruction, the posterior mediastinal route was used in $45(90 \%)$ patients, the subcutaneous route was used in four (8\%) patients, and the retrosternal route was used in one. Three-field lymph node dissection was performed in all patients. The median number of dissected lymph nodes was 49.5 (range $=21-92$ ). The median hospital stay was 27.5 (range $=16-101)$ days, and no patients died in the hospital or within 90 days after surgery.

The pathological findings for patients receiving NACRT followed by esophagectomy are shown in Table I. Among the 50 patients, 13 (26\%) were pT0, 28 (56\%) were pN0, and $10(20 \%)$ showed a pCR (CR in both tumor and lymph nodes). The histological responses to NACRT were grade 3 in 13 (26\%) patients and grade 2-3 in $36(72 \%)$ patients.

The median observation period was 43.5 (range=6-94) months after initial treatment. Disease recurred in 24 (48\%) patients; among these, 21 (88\%) recurrences were distant metastasis (17 in organs and four in lymph nodes outside the surgical field), and the remaining three (13\%) were local 
Table I. Patient backgrounds and pathological findings.

\begin{tabular}{|c|c|}
\hline & Value \\
\hline Cases, $\mathrm{n}$ & 50 \\
\hline Age, years & $62.5(43-74)$ \\
\hline \multicolumn{2}{|l|}{ Gender, n (\%) } \\
\hline Male & $41(82 \%)$ \\
\hline Female & $9(18 \%)$ \\
\hline \multicolumn{2}{|l|}{ Tumor location, n (\%) } \\
\hline Upper & $12(24 \%)$ \\
\hline Middle & $23(46 \%)$ \\
\hline Lower, abdominal & $15(30 \%)$ \\
\hline \multicolumn{2}{|l|}{$\mathrm{cT}, \mathrm{n}(\%)$} \\
\hline $\mathrm{T} 1 \mathrm{~b}$ & $2(4 \%)$ \\
\hline $\mathrm{T} 2$ & $4(8 \%)$ \\
\hline $\mathrm{T} 3$ & $43(86 \%)$ \\
\hline $\mathrm{T} 4$ & $1(2 \%)$ \\
\hline \multicolumn{2}{|l|}{$\mathrm{cN}$} \\
\hline N0, n $(\%)$ & $0(0 \%)$ \\
\hline $\mathrm{N} 1, \mathrm{n}(\%)$ & $32(64 \%)$ \\
\hline $\mathrm{N} 2, \mathrm{n}(\%)$ & $17(34 \%)$ \\
\hline $\mathrm{N} 3, \mathrm{n}(\%)$ & $1(2 \%)$ \\
\hline $\begin{array}{l}\text { Median number of involved LNs (range) } \\
\mathrm{cM}, \mathrm{n}(\%)\end{array}$ & $2(1-7)$ \\
\hline M0 & $44(88 \%)$ \\
\hline M1 (distant LNs) & $6(12 \%)$ \\
\hline \multicolumn{2}{|l|}{ cStage, $\mathrm{n}(\%)$} \\
\hline IIB & $6(12 \%)$ \\
\hline IIIA & $20(40 \%)$ \\
\hline IIIB & $16(32 \%)$ \\
\hline IIIC & $2(4 \%)$ \\
\hline IV & $6(12 \%)$ \\
\hline \multicolumn{2}{|l|}{$\mathrm{pT}, \mathrm{n}(\%)$} \\
\hline T0 & $13(26 \%)$ \\
\hline $\mathrm{T} 1$ & $8(16 \%)$ \\
\hline $\mathrm{T} 2$ & $7(14 \%)$ \\
\hline $\mathrm{T} 3$ & $21(42 \%)$ \\
\hline $\mathrm{T} 4$ & $1(2 \%)$ \\
\hline \multicolumn{2}{|l|}{$\mathrm{pN}$} \\
\hline N0, n $(\%)$ & $28(56 \%)$ \\
\hline $\mathrm{N} 1, \mathrm{n}(\%)$ & $14(28 \%)$ \\
\hline $\mathrm{N} 2, \mathrm{n}(\%)$ & $8(16 \%)$ \\
\hline Median number of involved LNs (range) & $0(0-4)$ \\
\hline pCR in both tumor and LNs, $\mathrm{n}(\%)$ & $10(20 \%)$ \\
\hline \multicolumn{2}{|l|}{ Histological CRT response in the tumor, $\mathrm{n}(\%)$} \\
\hline Grade 3 & $13(26 \%)$ \\
\hline Grade 2 & $23(46 \%)$ \\
\hline Grade 1 & $14(28 \%)$ \\
\hline
\end{tabular}

LNs: Lymph nodes, CRT: chemoradiotherapy, pCR: pathological complete response.

(two in lymph nodes within the surgical field and one intramural within the residual esophagus). The 2-year and 3year overall survival rates were $88 \%$ and $72 \%$, respectively, and the disease-specific survival rates were $90 \%$ and $73 \%$, respectively.
Table II. Relationship of the fractional decrease in maximum standardized uptake value $\left(S U V_{\max }\right)$ in $\left[^{18} \mathrm{~F}\right]$-fluorodeoxyglucose positron-emission tomography/computed tomography with pathological complete response $(p C R)$ and recurrence.

\begin{tabular}{lccc}
\hline & \multicolumn{3}{c}{$\mathrm{SUV}_{\max }, \mathrm{n}$} \\
\cline { 2 - 3 } Status & Decrease $<75 \%$ & Decrease $\geq 75 \%$ & \\
\hline pCR & 1 & 6 & 0.0918 \\
Non-pCR & 16 & 13 & \\
Recurrence & 13 & 7 & $0.0228^{*}$ \\
Non-recurrence & 4 & 12 & \\
\hline
\end{tabular}

*Significant difference.

All 42 patients examined were found to be positive for tumors on FDG-PET/CT before treatment. After NACRT, $28 \%(10 / 36)$ of patients had changed from positive to negative for tumor on FDG-PET/CT. Seventy-one percent (30/42) of patients were positive for lymph node involvement on FDG-PET/CT before treatment. After NACRT, $81 \%$ (22/27) of patients previously positive for lymph node involvement were negative on FDG-PET/CT. The median fractional decrease in tumor $\mathrm{SUV}_{\max }$ was $75 \%$ (range $=19-90 \%$ ). We therefore divided the patients into two groups, with a decrease in tumor $\mathrm{SUV}_{\max }$ of $<75 \%$ or $\geq 75 \%$, and investigated the relationship of the decrease in tumor $\mathrm{SUV}_{\max }$ with pCR and recurrence (Table II). We found that there was a significant correlation between a decrease in FDG-PET/CT and recurrence but not pCR.

When we compared the survival rates between grade 2-3 responders to NACRT and grade 1 non-responders, we found significantly better survival among the responders $(p=0.0311)$. The 2- and 3-year overall survival rates among grade 2-3 responders to NACRT were $94 \%$ and $78 \%$, respectively (Figure 1). When we compared the survival rates according to fractional decrease in tumor $\mathrm{SUV}_{\max }$, we found significantly better survival among the metabolic responders ( $\left.\mathrm{SUV}_{\max } \geq 75 \%, p=0.0375\right)$. The 2- and 3-year overall survival rates among metabolic responders to NACRT were $89 \%$ and $79 \%$, respectively (Figure 1).

Consistent with those findings, univariate analysis taking into consideration age, gender, $\mathrm{cT}, \mathrm{cN}, \mathrm{cM}$, adverse events, interval between NACRT and surgery, number of dissected LNs, pT, pN, pCR, pathological response, and fractional decrease in tumor $\mathrm{SUV}_{\max }$ showed that age ( $\geq 63$ years), gender (male), cT (T3-4), grade 1 pathological response and fractional decrease in tumor $\mathrm{SUV}_{\max }$ of $<75 \%$ to be significant prognostic factors associated with poorer survival (Table III). A subsequent multivariate analysis taking these factors into consideration showed decreased tumor SUV $\mathrm{Sax}_{\text {max }}$ and gender to be significant prognostic factors in patients with esophageal 

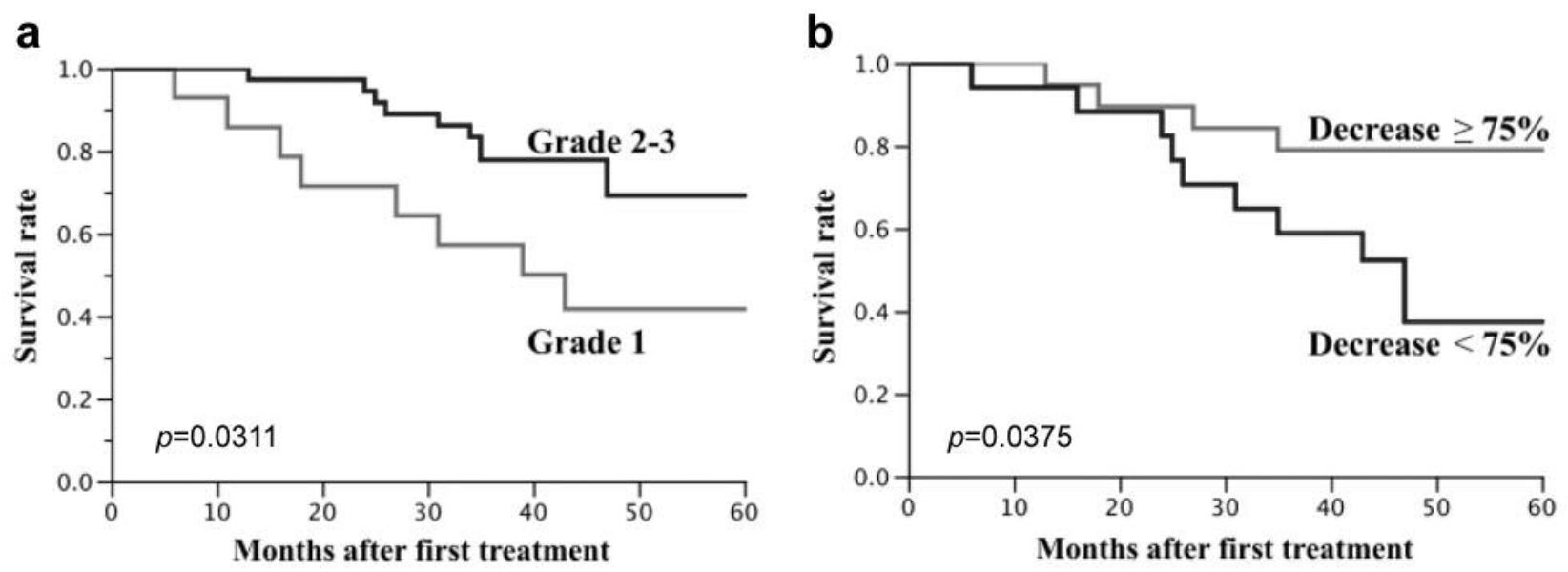

Figure 1. Comparison of overall survival rates according to pathological grade response (a) and fractional decrease in tumor maximum standardized uptake value on $\left.{ }^{18} \mathrm{~F}\right]$-fluorodeoxyglucose positron-emission tomography/computed tomography $(b)$. Note the significant difference between the two groups.

Table III. Univariate and multivariate analyses of 5-year overall survival in the Cox proportional hazard regression model.

\begin{tabular}{|c|c|c|c|c|c|c|c|}
\hline & & \multicolumn{3}{|c|}{ Univariate analysis } & \multicolumn{3}{|c|}{ Multiivariate analysis } \\
\hline & & Hazard ratio & $95 \% \mathrm{CI}$ & $p$-Value & Hazard ratio & $95 \% \mathrm{CI}$ & $p$-Value \\
\hline Age & $\geq 63$ vs. $<63$ years & 2.638 & $1.0131-7.6577$ & $0.0469^{*}$ & - & $0.6276-6.5206$ & 0.2569 \\
\hline Gender & Male $v s$. female & $\infty$ & $2.3869-2.3869$ & $0.0343^{*}$ & - & $1.1425-\infty$ & $0.0375^{*}$ \\
\hline $\mathrm{cT}$ & T3-4 vs. T1-2 & $\infty$ & $1.1774-1.1774$ & $0.0343^{*}$ & - & $0.6971-\infty$ & 0.0905 \\
\hline $\mathrm{cN}$ & $\mathrm{N} 2-3$ vs. N1 & - & $0.5264-3.5035$ & 0.5008 & & & \\
\hline $\mathrm{cM}$ & M1 vs. M0 & - & $0.3084-4.0388$ & 0.6611 & & & \\
\hline Grade $>3$ adverse events & Yes vs. no & - & $0.4374-3.0281$ & 0.8251 & & & \\
\hline Interval between NACRT and Surgery & $<6 v s . \geq 6$ Weeks & - & $0.3342-2.5016$ & 0.7778 & & & \\
\hline Number of dissected LNs & $<50 v s . \geq 50$ & - & $0.5671-3.7703$ & 0.4418 & & & \\
\hline pT & $\mathrm{T} 2-4 v s . \mathrm{T} 0-1$ & - & $0.6282-4.6624$ & 0.3242 & & & \\
\hline $\mathrm{pN}$ & N1-3 vs. N0 & - & $0.4363-2.9000$ & 0.8038 & & & \\
\hline $\mathrm{pCR}$ & Non-pCR vs. pCR & - & $0.89837-9.073$ & 0.0721 & & & \\
\hline Pathological response & Grade 1 vs. 2-3 & 2.663 & $1.0154-6.7608$ & $0.0467 *$ & - & $0.7119-6.8931$ & 0.1597 \\
\hline Fractional decrease in tumor $\mathrm{SUV}_{\max }$ & $<75 \%$ vs. $\geq 75 \%$ & 3.180 & $1.06271-1.601$ & $0.0382^{*}$ & 3.169 & $1.0561-11.582$ & $0.0393 *$ \\
\hline
\end{tabular}

NACRT: neoadjuvant chemoradiotherapy, LN: lymph node, $\mathrm{SUV}_{\max }:$ maximum standardized uptake value. $*$ Significant difference. $\infty$ Estimated values were infinitely large.

cancer receiving NACRT plus esophagectomy, on the other hand, cT, cN, pT and pN were not. These findings suggest that metabolic response could be a stronger predictor of 3-year survival compared to pathological response in patients receiving NACRT plus esophagectomy.

\section{Discussion}

In this study, we demonstrated that 3-year overall survival rates in grade 2-3 pathological responders or metabolic responders to NACRT having a fractional decrease in tumor
$\mathrm{SUV}_{\text {max }} \geq 75 \%$, were $78 \%$ and $79 \%$, respectively. Metabolic response as a decrease in $\mathrm{SUV}_{\max }$ of $\geq 75 \%$ was an independent prognostic factor for 3-year overall survival in multivariate analysis and associated with recurrence. On the other hand, cT, cN, pT, pN, pCR were not associated with survival outcome or recurrence.

In the CROSS trial, a multicenter randomized controlled trial started in 2004, the 5-year overall survival in the NACRT-treated group $(n=178)$ was $47 \%$, while that in the group treated with surgery alone $(n=188)$ was $33 \%$ (4). The latest randomized control trial to compare NAC 
with NACRT for cancer of the esophagus or esophagogastric junction detected no difference in 3-year overall survival in the two treatment groups (NAC, $49 \% \mathrm{vs}$. NACRT, 47\%) (8).

NACRT plus esophagectomy is a powerful treatment but is only effective in patients with tumor sensitivity to NACRT. Therefore, a reliable marker that is predictive of the susceptibility to NACRT is being sought. Swisher et al. reported that 3 -year survival rates were significantly higher among patients who had smaller remnant tumors on postoperative histopathological examination (9). In addition, Law et al. showed that pN0 and R0 were statistically significant factors predictive of a better prognosis (10). Moreover, according to Schneider et al. (11) and Okumura et al. (12) prognosis was significantly better among patients with shrunken tumors or with tumors classified as pN0. Furthermore, Ohkura et al. recently reported that survival rates were increased when there was down-staging due to tumor shrinkage and grade 2 or greater treatment efficacy based on the histological grading system (13). These studies reported that pathological response was a useful predictor for survival outcome, but these were for postoperative factors and therefore not prescriptive. Consequently, a useful preoperative clinical predictive factor is necessary.

Song et al. reported that the sensitivity, specificity, and accuracy of FDG-PET findings of complete metabolic remission after NACRT were $27 \%, 95 \%$ and $72 \%$, respectively, when predicting $\mathrm{pCR}$ (14). The correlation between a complete metabolic response after NACRT and pCR in TESCC was $71 \%$ for accuracy (15). Hamai et al. recently reported that the fractional decrease in the tumor on FDG-PET/CT is a valuable predictor of the pathological response to NACRT and survival. They also showed that the optimal cutoff for a decrease predictive of pCR is $75 \%$. Furthermore, in a multivariate analysis, fractional decrease in SUV $_{\text {max }}$ using a out off of $70 \%$ was an independent prognostic factor associated with better disease-specific survival $(\mathrm{HR}=0.45 ; 95 \% \mathrm{CI}=0.21-0.98 ; p=0.04)(16)$. Park et al. reported that FDG-PET has 73\% sensitivity, 86\% specificity and $80 \%$ accuracy for pCR prediction after CRT for TESCC when the optimal cutoff for a decrease in $\mathrm{SUV}_{\max }$ on FDG-PET/CT after NACRT was $72 \%$ (17). These findings indicate that the magnitude of the tumor metabolic response determined with preoperative FDG-PET is an important preoperative prognostic indicator for patients undergoing trimodal therapy, and that the magnitude of the difference between the metabolic activity in a primary tumor before and after NACRT is a useful guide for postoperative therapy and for establishing postoperative surveillance programs. However, there is still no consensus as to the optimal time points, evaluation methods, or cut-off values for metabolic responses determined by preoperative FDG-PET.
Not all patients treated with NACRT ultimately undergo the scheduled surgery. The surgery may be cancelled due to a worsening of the patient's general condition or the patient my refuse the surgery due to the negative impact on quality of life after esophagectomy (18). Refusal to undergo surgery may be particularly common in the subgroup of patients who show a good response to NACRT. However, Fang et al. reported that at least $60 \%$ of patients with TESCC who achieved clinical CR following NACRT but subsequently refused the planned esophagectomy experienced disease recurrence, with local recurrence being the predominant failure pattern (19). Surgeons should be aware of this fact and inform their patients of the risk of recurrence after NACRT.

In conclusion, metabolic response to NACRT was an independent prognostic factor and stronger predictor for survival and recurrence compare to pathological response in patients with cStage IIB-IV TESCC.

\section{Acknowledgements}

This work was supported, in part, by Grants-in-Aid for Scientific Research from the Ministry of Education, Culture, Science, Sports and Technology-Japan.

\section{References}

1 Rustgi AK and El-Serag HB: Esophageal carcinoma. N Engl J Med 371(26): 2499-2509, 2014.

2 Wijnhoven BP, van Lanschot JJ, Tilanus HW, Steyerberg EW and van der Gaast A: Neoadjuvant chemoradiotherapy for esophageal cancer: a review of meta-analyses. World J Surg 33(12): 2606-2614, 2009.

3 Deng J, Wang C, Xiang M, Liu F, Liu Y and Zhao K: Metaanalysis of postoperative efficacy in patients receiving chemoradiotherapy followed by surgery for resectable esophageal carcinoma. Diagn Pathol 9: 151, 2014.

4 Shapiro J, van Lanschot JJ, Hulshof MC, van Hagen P, van Berge Henegouwen MI, Wijnhoven BP, van Laarhoven HW, Nieuwenhuijzen GA, Hospers GA, Bonenkamp JJ, Cuesta MA, Blaisse RJ, Busch OR, Ten Kate FJ, Creemers GJ, Punt CJ, Plukker JT, Verheul HM, Bilgen EJ, van Dekken H, van der Sangen MJ, Rozema T, Biermann K, Beukema JC, Piet AH, van Rij CM, Reinders JG, Tilanus HW, Steyerberg EW and van der Gaast A; CROSS study group: Neoadjuvant chemoradiotherapy plus surgery versus surgery alone for oesophageal or junctional cancer (CROSS): long-term results of a randomized controlled trial. Lancet Oncol 16(9): 1090-1098, 2015.

5 UICC - International Union Against Cancer. TNM Classification of Malignant Tumours, Seventh Edition. Sobin LH, Gospodarowicz MK and Wittekind CH (eds.). Wiley-Blackwell, A John Wiley \& Sons, Ltd, 2009.

6 Japan Esophageal Society: Japanese Classification of Esophageal Cancer, 11th Edition: part I. Esophagus 14(1): 1-36, 2017.

7 Japan Esophageal Society: Japanese Classification of Esophageal Cancer, 11th Edition: part II and III. Esophagus 14(1): 37-65, 2017. 
8 Klevebro F, Alexandersson von Dobeln G, Wang N, Johnsen G, Jacobsen AB, Friesland S, Hatlevoll I, Glenjen NI, Lind P, Tsai JA, Lundell L and Nilsson M: A randomized clinical trial of neoadjuvant chemotherapy $v s$. neoadjuvant chemoradiotherapy for cancer of the oesophagus or gastro-oesophageal junction. Ann Oncol 27(4): 660-667, 2016.

9 Law S, Kwong DL, Wong KH, Kwok KF and Wong J: The effects of neoadjuvant chemoradiation on pTNM staging and its prognostic significance in esophageal cancer. J Gastrointest Surg 10(9): 1301-1311, 2006.

10 Naunheim KS, Petruska P, Roy TS, Andrus CH, Johnson FE, Schlueter JM and Baue AE: Preoperative chemotherapy and radiotherapy for esophageal carcinoma. $\mathrm{J}$ Thorac Cardiovasc Surg 103(5): 887-893, 1992.

11 Schneider PM, Baldus SE, Metzger R, Kocher M, Bongartz R, Bollschweiler E, Schaefer H, Thiele J, Dienes HP, Mueller RP and Hoelscher AH: Histomorphologic tumor regression and lymph node metastases determine prognosis following neoadjuvant radiochemotherapy for esophageal cancer: implications for response classification. Ann Surg 242(5): 684-692, 2005.

12 Okumura H, Uchikado Y, Matsumoto M, Owaki T, Kita Y, Omoto I, Sasaki K, Sakurai T, Setoyama T, Nabeki B, Matsushita D, Ishigami S, Hiraki Y and Nakajo M: Prognostic factors in esophageal squamous cell carcinoma patients treated with neoadjuvant chemoradiation therapy. Int $\mathrm{J}$ Clin Oncol 18(2): 329-334, 2013.

13 Ohkura Y, Ueno M, Iizuka T, Haruta S, Tanaka T and Udagawa $\mathrm{H}$ : Factors predicting effectiveness of neoadjuvant therapy for esophageal squamous cell carcinoma. Medicine 95(15): e3365, 2016.

14 Song SY, Kim JH, Ryu JS, Lee GH, Kim SB, Park SI, Song HY, Cho KJ, Ahn SD, Lee SW, Shin SS and Choi EK: FDG-PET in the prediction of pathologic response after neoadjuvant chemoradiotherapy in locally advanced, resectable esophageal cancer. Int J Radiat Oncol Biol Phys 63(4): 1053-1059, 2005.
15 Kim MK, Ryu JS, Kim SB, Ahn JH, Kim SY, Park SI, Kim YH, Song HY, Shin JH, Jung HY, Lee GH, Choi KD, Cho KJ and Kim JH: Value of complete metabolic response by (18)Ffluorodeoxyglucose positron-emission tomography in oesophageal cancer for prediction of pathologic response and survival after preoperativechemoradiotherapy. Eur J Cancer 43(9): 1385-1391, 2007.

16 Hamai Y, Hihara J, Emi M, Furukawa T, Yamakita I, Kurokawa $\mathrm{T}$ and Okada M: Ability of fluorine-18 fluorodeoxyglucose positron-emission tomography to predict outcomes of neoadjuvant chemoradiotherapy followed by surgical treatment for esophageal squamous cell carcinoma. Ann Thorac Surg 102(4): 1132-1139, 2016.

17 Park JS, Choi JY, Moon SH, Ahn YC, Lee J, Kim D, Kim K and Shim YM: Response evaluation after neoadjuvant chemoradiation by positron-emission tomography-computed tomography for esophageal squamous cell carcinoma. Cancer Res Treat 45(1): 22-30, 2013.

18 Scarpa M, Valente S, Alfieri R, Cagol M, Diamantis G, Ancona $\mathrm{E}$ and Castoro C: Systematic review of health-related quality of life after esophagectomy for esophageal cancer. World J Gastroenterol 17(42): 4660-4674, 2011.

19 Fang HY, Chao YK, Chang HK, Tseng CK and Liu YH: Survival outcomes of consolidation chemoradiotherapy in esophageal cancer patients who achieve clinical complete response but refuse surgery after neoadjuvant chemoradiotherapy. Dis Esophagus 30(2): 1-8, 2017. 